\title{
ON THE VARIATIONAL METHOD FOR THE EXISTENCE OF SOLUTIONS OF NONLINEAR EQUATIONS OF HAMMERSTEIN TYPE
}

\author{
DJAIRO G. DEFIGUEIREDO AND CHAITAN P. GUPTA ${ }^{1}$
}

\begin{abstract}
Let $X$ be a real Banach space and $X^{*}$ its conjugate Banach space. Let $A$ be an unbounded monotone linear mapping from $X$ to $X^{*}$ and $N$ a potential mapping from $X^{*}$ to $X$. In this paper we establish the existence of a solution of the equation $u+A N u=v$ for a given $v$ in $X^{*}$ using variational method. Our method consists in using a splitting of $A$ via an auxiliary Hilbert space and solving an equivalent equation in this auxiliary Hilbert space. In $\$ 2$, we prove the same result in the case when $X$ is a Hilbert space using the natural splitting of $A$ in terms of its square root. We do this to compare and contrast the proofs in the two cases.
\end{abstract}

Introduction. Let $X$ be a real Banach space and $X^{*}$ denote its conjugate Banach space. Let $A$ be an unbounded monotone linear mapping from $X$ to $X^{*}$ and $N$ a nonlinear mapping from $X^{*}$ to $X$ satisfying no monotone hypothesis. In this paper we study the solvability of the equation

$$
u+A N u=v
$$

for a given element $v$ in $X^{*}$. Equations of the form given in (1) are known as equations of Hammerstein type. The most interesting example of (1) is when $A$ is a linear integral mapping and $N$ is a so-called Nemitsky mapping. The case when $A$ is an unbounded linear mapping corresponds to the case of linear integral mappings having Carleman kernels. We may mention that our study of equation (1) when $A$ is an unbounded linear mapping makes it possible to apply our results not only to nonlinear integral equations of Hammerstein type but also to certain boundary value problems in nonlinear differential equations.

In recent years there has been extensive work on these equations under various monotonicity assumptions on $N$. A recent paper of Browder [1] contains a fairly complete bibliography on this subject. The authors in their previous works [3], [4] had considered the case when the linear

Received by the editors April 7, 1971.

AMS (MOS) subject classifications (1970). Primary 47H15, 49G99, 45G05, 45G99.

Key words and phrases. Variational method, integral equations of Hammerstein type, weak*-gradient, Carleman kernels.

${ }^{1}$ Part of the research of this author supported by NSF grant \#GP28735.

c American Mathematical Society 1973 
mapping $A$ is unbounded and $N$ satisfied some sort of monotonicity conditions. In case $N$ does not satisfy any monotonicity condition it seems appropriate to use variational methods. However, one is bound to have several difficulties stemming from the unbounded nature of the linear mapping $A$. In a Hilbert space these difficulties could be handled because the unbounded monotone linear mapping $A$ has a natural splitting in terms of its square root, while no such thing had been known in the case of Banach spaces. It is for these reasons (it seems) that Vainberg and Lavrentiev [8] in their joint work impose several extraneous conditions on the Banach spaces and on the linear mapping $A$, for then they are able to reduce equation (1) to an equivalent equation in a Hilbert space and use the known splitting there. In this paper, we have been able to study (1) without any such restrictions by using a new splitting of $A$ and our results generalize their results.

A subset $D$ of $X \times X^{*}$ is said to be monotone if $\left(x-y, x^{*}-y^{*}\right) \geqq 0$ for all pairs $\left[x, x^{*}\right],\left[y, y^{*}\right]$ in $D$. Here $\left(x, x^{*}\right)$ denotes the duality pairing between the elements $x$ of $X$ and $x^{*}$ of $X^{*}$ and in the following we make the convention that $\left(x^{*}, x\right)=\left(x, x^{*}\right)$. A monotone set is said to be maximal monotone if it is not a proper subset of any other monotone set. A mapping $T: D(T) \rightarrow X^{*}$, where $D(T) \subset X$, is monotone if its graph $G(T)=$ $\{[x, T x]: x \in D(T)\}$ is a monotone set, and $T$ is maximal monotone if its graph is a maximal monotone set. A linear mapping $A: D(A) \rightarrow X^{*}, D(A) \subset X$, is symmetric if $(A x, y)=(A y, x)$ for every $x, y$ in $D(A)$. A mapping $N: X^{*} \rightarrow X$ is called a weak ${ }^{*}$-gradient if there exists a functional $f: X^{*} \rightarrow \boldsymbol{R}$ (where $\boldsymbol{R}$ denotes the set of real numbers) such that

$$
\left(N x^{*}, y^{*}\right)=\lim _{t \rightarrow 0} \frac{f\left(x^{*}+t y^{*}\right)-f\left(x^{*}\right)}{t}
$$

for every $x^{*}, y^{*}$ in $X^{*}$. In particular, if $X$ is reflexive the notion of weak*gradient coincides with that of weak-gradient. We remark that if $X$ is not reflexive and a given $f: X^{*} \rightarrow R$ is weakly-Gateaux differentiable then its weak-gradient is a mapping from $X^{*}$ to $X^{* *}$ and so $f$ does not give rise, in general, to a mapping of the type considered here.

Our main result is as follows:

THEOREM 1. Let $X$ be a real Banach space such that the unit ball in its conjugate $X^{*}$ is neak*-sequentially-compact. (In particular, this is the case if $X$ is either reflexive or separable.) Let $A: D(A) \rightarrow X^{*}, D(A) \subset X$, be a linear maximal monotone symmetric densely defined mapping from $X$ to $X^{*}$. Let $N: X^{*} \rightarrow X$ be the weak*-gradient of a functional $f: X^{*} \rightarrow \boldsymbol{R}$ which is assumed to be lower-weak*-semicontinuous and such that

$$
f\left(x^{*}\right) \geqq(1)\left(\left\|x^{*}\right\|\right) \text { for all } x^{*} \text { in } X^{*}
$$


where the numerical function $\omega(r)$ is bounded below for $r \geqq 0$ and $\omega(r) \rightarrow+\infty$ as $r \rightarrow+\infty$. Then, there exists $x^{*} \in X^{*}$ such that $x^{*}+A N x^{*}=y^{*}$ for each given $y^{*}$ in $X^{*}$.

Remarks. (i) The bound (2) on $f$ required in Theorem 1 is satisfied if

$$
f\left(x^{*}\right) \geqq a_{1}\left\|x^{*}\right\|^{2}+a_{2}\left\|x^{*}\right\|^{\theta}+a_{3}
$$

where $a_{1}>0, a_{2}$ and $a_{3}$ are any given real numbers, $0<0<2$. The estimate (3) arises in several situations for the potential of Nemitsky mappings, see [9].

(ii) Compare the above theorem with Theorem 4 of [8] where even under more stringent conditions only a sort of generalized solution is obtained.

(iii) Without loss of generality we may assume that $y^{*}=0$. For if this is not the case we replace $N$ by the mapping $N_{1}$ defined by $N_{1}\left(x^{*}\right)=$ $N\left(x^{*}+y^{*}\right)$ and it is easy to verify that $N_{1}$ satisfies the conditions of Theorem 1, above.

In the proof of Theorem 1, we use a splitting of the unbounded linear mapping $A$, which is given in Theorem 2, below. To obtain this splitting we follow Browder-Gupta [2] who considered the case of bounded $A$. We may also mention that Theorem 1, above, extends a previous result of Gupta [5] for the case of $A$ bounded.

THEOREM 2. Let $A: D(A) \rightarrow X^{*}$ be a linear monotone symmetric densely defined mapping from $X$ to $X^{*}$. Then there exists a Hilbert space $H, a$ linear mapping $S: D(A) \rightarrow H$ such that $A=S^{*} S$, where $S^{*}: D\left(S^{*}\right) \rightarrow X^{*}$, $D\left(S^{*}\right) \subset H$, is the adjoint of $S$.

The proofs of above theorems are given in $\S \S 1$ and 2 . In $\S 3$, we consider the Hilbert space case, where unlike the Banach space case we succeed in reducing the variational problem to the one of minimizing a functional on a complete set. In this manner we obtain some results which are comparable with results announced by Lavrentiev in a Doklady note [7].

1. Proof of Theorem 2. Consider in $D(A)$ the following bilinear form, $b[x, y]=(A x, y)$, which in view of the monotonicity of $A$ is such that $b[x, x] \geqq 0$. Consequently one has Cauchy-Schwarz inequality

$$
|b[x, y]| \leqq(b[x, x])^{1 / 2}(b[y, y])^{1 / 2} .
$$

Next we define the set $A=\{x \in D(A): b[x, x]=0\}$, which in view of (4) is also given by

$$
\mathscr{N}=\{x \in D(A): b[x, y]=0 \text { for all } y \text { in } D(A)\} .
$$


Thus $\mathscr{N}$ is a closed linear subspace of $D(A)$. So the quotient space $H_{0}=$ $D(A) / \mathscr{N}$ is a prehilbertian space with our inner product [, ] defined as follows. Let $\pi: D(A) \rightarrow H_{0}$ be the canonical mapping, and let $\bar{x}, \bar{y} \in H_{0}$, Then $[\bar{x}, \bar{y}]=(A x, y)$ where $x \in \pi^{-1}(\bar{x})$ and $y \in \pi^{-1}(\bar{y})$.

Now let us denote by $H$ the completion of $H_{0}$ with respect to this inner product. Let us finally denote by $S$ the composition of $\pi$ with the embedding mapping of $H_{0}$ into $H$ and let us also use [, ] for the inner product in $H$.

So the mapping $S: D(A) \rightarrow H$ is densely defined and has a dense range, by construction. It then follows that the adjoint mapping $S^{*}: D\left(S^{*}\right) \rightarrow X^{*}$ is injective. Now, we claim that $R(S) \subset D\left(S^{*}\right)$, which implies, in particular, that $S^{*}$ is densely defined. To prove the claimed inclusion let $S x \in R(S)$, $x \in D(A)$; the linear functional $l: D(A) \rightarrow \boldsymbol{R}$ defined by $l(y)=[S x, S y]$ is bounded because

$$
|[S x, S y]|=|(A x, y)| \leqq\|A x\| \cdot\|y\| .
$$

So $l$ can be extended to a bounded linear functional on $X$, which proves that $S(x) \in D\left(S^{*}\right)$ and $[S x, S y]=\left(S^{*} S x, y\right)$ for all $y$ in $D(A)$. On the other hand, $[S x, S y]=(A x, y)$, by definition. It then follows from the density of $D(A)$ in $X$ that $S^{*} S=A$, as we wanted to prove. Q.E.D.

2. Proof of Theorem 1. Let us denote the norm in $H$ by $|\cdot|$, and let us define a functional $\Phi: D(\Phi) \rightarrow R, D(\Phi) \subset H$, by

$$
\Phi(u)=\frac{1}{2}|u|^{2}+f\left(S^{*} u\right)
$$

where $S^{*}$ is the adjoint of the mapping $S$ defined in Theorem 2. So $D(\Phi)=D\left(S^{*}\right)$. In view of the hypothesis on $f$ we obtain $\Phi(u) \geqq \frac{1}{2}|u|^{2}+$ $\omega\left(\left\|S^{*} u\right\|\right)$. Now let $M=\inf \{\omega(r): r \geqq 0\}$. Thus $\Phi(u) \geqq \frac{1}{2}|u|^{2}+M$ for all $u \in D(\Phi)$ and the functional $\Phi$ is bounded from below on $D(\Phi)$. Let

$$
d=\inf \{\Phi(u): u \in D(\Phi)\}
$$

and let $\left\{u_{n}\right\}$ be a minimizing sequence for $\Phi$. It is clear that, for all $n$ sufficiently large,

$$
d+1 \geqq \Phi\left(u_{n}\right) \geqq \frac{1}{2}\left|u_{n}\right|^{2}+\omega\left(\left\|S^{*} u_{n}\right\|\right)>M .
$$

It then follows from (6) that, the sequences $\left\{u_{n}\right\}$ and $\left\{S^{*} u_{n}\right\}$ are bounded. In view of the weak compactness of the ball in $H$ and the weak*-sequential compactness of the ball in $X^{*}$ we may assume (by going to a subsequence, if necessary) that there exist elements $u_{0}$ in $H$ and $y^{*}$ in $X^{*}$ such that $u_{n} \rightarrow u_{0}$ in $H$ and $S^{*} u_{n} \rightarrow^{*} y^{*}$ in $X^{*}$, where $\rightarrow$ denotes the weak convergence and $\rightarrow^{*}$ weak* convergence. Now we claim that $u_{0} \in D\left(S^{*}\right)$. Indeed, all we have to do is to prove that the linear functional $l: D(A) \rightarrow R$ defined 
by $l(x)=\left[u_{0}, S x\right]$ is bounded. And this is so because

$$
\begin{aligned}
{\left[u_{0}, S x\right] } & =\lim \left[u_{n}, S x\right]=\lim \left(S^{*} u_{n}, x\right)=\left(y^{*}, x\right), \text { and } \\
\left|\left[u_{0}, S x\right]\right| & \leqq\left\|y^{*}\right\| \cdot\|x\| ;
\end{aligned}
$$

from (7) it follows that $S^{*} u_{0}=y^{*}$. Next we prove that $\Phi(u)$ assumes its infimum at $u_{0}$, i.e., $\Phi\left(u_{0}\right)=d$. Indeed, since the norm in $H$ is weakly-lowersemicontinuous and $f$ is weak*-lower-semicontinuous we obtain that

$$
\frac{1}{2}\left|u_{0}\right|^{2}+f\left(S^{*} u_{0}\right) \leqq \lim \inf \left\{\frac{1}{2}\left|u_{n}\right|^{2}+f\left(S^{*} u_{n}\right)\right\}=\lim \Phi\left(u_{n}\right)=d .
$$
have

Now, since $\Phi$ has a minimum at $u_{0}$, it follows that for all $u \in D(\Phi)$, we

$$
\lim _{t \rightarrow 0} \frac{\Phi\left(u_{0}+t u\right)-\Phi\left(u_{0}\right)}{t}=0
$$

when such a limit exists. It is, however, easy to see that this limit exists for all $u \in D(\Phi)$, and we obtain

$$
\left[u_{0}, u\right]+\left(N\left(S^{*} u_{0}\right), S^{*} u\right)=0 .
$$

We, now, claim that $N\left(S^{*} u_{0}\right) \in D(A)$ and, moreover, $A N S^{*} u_{0}=-S^{*} u_{0}$. This we can do if we prove that

$$
\left(A x+S^{*} u_{0}, x-N S^{*} u_{0}\right) \geqq 0
$$

for all $x \in D(A)$, in view of the maximal monotonicity of $A$. Using (8) first with $u=u_{0}$ and then with $\imath=S x$ we obtain

$$
-\left(N\left(S^{*} u_{0}\right), S^{*} u_{0}\right)=\left[u_{0}, u_{0}\right], \quad-\left(N\left(S^{*} u_{0}\right), S^{*} S x\right)=\left[u_{0}, S x\right] .
$$

This reduces (9) to the inequality

$$
(A x, x)+\left(S^{*} u_{0}, x\right)+\left[u_{0}, u_{0}\right]+\left[u_{0}, S x\right] \geqq 0 .
$$

Now let $\left\{x_{n}\right\} \subset D(A)$ be such that $S x_{n} \rightarrow u_{0}$ in $H$. It is then easy to see that (10) is obtained from the inequality $\left(A x+A x_{n}, x+x_{n}\right)=\left|S x+S x_{n}\right|^{2} \geqq$ 0 by taking the limit as $n \rightarrow \infty$.

Finally, from the relation $S^{*} u_{0}+A N S^{*} u_{0}=0$ it follows that $x^{*}=S^{*} u_{0}$ is a solution of $x^{*}+A N x^{*}=0$. Q.E.D.

3. Equations in a Hilbert space. We begin with some remarks on selfadjoint (not necessarily bounded) linear mappings in Hilbert spaces. If $A: D(A) \rightarrow H$, where $D(A)$ is a dense subspace of the Hilbert space $H$, is selfadjoint and monotone, then it follows that $A$ is closed, densely defined and maximal monotone. So $A$ is $m$-accretive in the sense of Kato [6]. Thus $A$ has a square $\operatorname{root} A^{1 / 2}$, which is also selfadjoint and $m$-accretive, see [6]. 
The next result is an immediate consequence of Theorem 1. However we include another proof in the hope that the ideas expounded there may be useful in other situations.

THEOREM 3. Let $A: D(A) \rightarrow H$ be a selfadjoint monotone linear mapping in a real Hilbert space $H$. Let $f: H \rightarrow R$ be a weakly Gateaux differentiable functional which is also weakly lower semicontinuous and is such that $f(x) \geqq$ $a_{1}\|x\|^{2}+a_{2}\|x\|^{\theta}+a_{3}$ where $a_{1}>0, a_{2} \leqq 0, a_{3} \leqq 0,0<\theta<2$. Let $N$ denote the weak-gradient of $f$.

Then the equation $x+A N x=y$, for a given $y$ in $H$, has at least one solution.

Proof. We can see as in the Remark (iii) after Theorem 1 that there is no loss of generality in assuming that $y=0$. Define in $D\left(A^{1 / 2}\right)$ the following inner-product $[x, y]=(x, y)+\left(A^{1 / 2} x, A^{1 / 2} y\right)$, which gives the norm $|x|=\left(\|x\|^{2}+\left\|A^{1 / 2} x\right\|^{2}\right)^{1 / 2}$.

The linear subspace $D\left(A^{1 / 2}\right)$ with this new norm is complete and consequently is a Hilbert space which we denote by $H^{\prime}$. In fact, if $\left\{u_{n}\right\}$ is a Cauchy sequence in $H^{\prime}$ then $\left\{u_{n}\right\}$ and $\left\{A^{1 / 2} u_{n}\right\}$ are both Cauchy sequences in $H$. So there are $u, v$ in $H$ such that $u_{n} \rightarrow u$ and $A^{1 / 2} u_{n} \rightarrow v$ in $H$. Since $A^{1 / 2}$ is closed we see that $u \in D\left(A^{1 / 2}\right)$ and $v=A^{1 / 2} u$. So $u \in H^{\prime}$ and clearly $u_{n} \rightarrow u$ in $H^{\prime}$.

Now, consider the functional $\Phi: H^{\prime} \rightarrow \boldsymbol{R}$ defined by $\Phi(u)=\frac{1}{2}\|u\|^{2}+$ $f\left(A^{1 / 2} u\right)$. We shall prove, now, that $\Phi$ is weakly-lower-semicontinuous in $H^{\prime}$. To do this we first prove that if $u_{n} \rightarrow u$ in $H^{\prime}$, then $u_{n} \rightarrow u$ in $H$ and $A^{1 / 2} u_{n} \rightarrow A^{1 / 2} u$ in $H$. In fact,

$$
\begin{aligned}
{\left[u_{n}, v\right] } & =\left(u_{n}, v\right)+\left(A^{1 / 2} u_{n}, A^{1 / 2} v\right) \\
& =\left(u_{n}, v+A v\right) \quad \text { for all } v \in D\left(A^{1 / 2}\right) .
\end{aligned}
$$

Since $D(A) \subset D\left(A^{1 / 2}\right)$ and $R(I+A)=H$ by virtue of $m$-accretiveness of $A$ it follows from (11) that $u_{n} \rightarrow u$ in $H$. Similarly, using the expression

$$
\left[u_{n}, A^{1 / 2} v\right]=\left(u_{n}, A^{1 / 2} v\right)+\left(A^{1 / 2} u_{n}, A v\right)=\left(A^{1 / 2} u_{n}, v+A v\right)
$$

we prove that $A^{1 / 2} u_{n} \rightarrow A^{1 / 2} u$ in $H$. Thus we have

$$
\|u\| \leqq \lim \inf \left\|u_{n}\right\|, \quad f\left(A^{1 / 2} u\right) \leqq \lim \inf f\left(A^{1 / 2} u_{n}\right),
$$

whenever $u_{n} \rightarrow u$ in $H^{\prime}$. These two inequalities then imply that $\Phi(u) \leqq$ $\lim \inf \Phi\left(u_{n}\right)$ whenever $u_{n} \rightarrow u$ in $H^{\prime}$. Thus $\Phi$ is weakly-lower-semicontinuous in $H^{\prime}$.

Moreover, from the assumptions on $f$ we have

$$
\begin{aligned}
\Phi(u) & \geqq \frac{1}{2}\|u\|^{2}+a_{1}\left\|A^{1 / 2} u\right\|^{2}+a_{2}\left\|A^{1 / 2} u\right\|^{\theta}+a_{3} \\
& \geqq \min \left(\frac{1}{2}, a_{1}\right)|u|^{2}+a_{2}\left\|A^{1 / 2} u\right\|^{\theta}+a_{3},
\end{aligned}
$$


which implies that $\Phi(u) \rightarrow+\infty$ as $|u| \rightarrow+\infty$. Thus there exists a $u_{0} \in H^{\prime}$ where $\Phi$ assumes a minimum. At this point the gradient of $\Phi$ vanishes and this gives

$$
\left(u_{0}, v\right)+\left(N A^{1 / 2} u_{0}, A^{1 / 2} v\right)=0 .
$$

Since this holds for all $v \in D\left(A^{1 / 2}\right)$ we obtain that $N A^{1 / 2} u_{0} \in D\left(A^{1 / 2}\right)$ and $A^{1 / 2} N A^{1 / 2} u_{0}=-u_{0}$. Finally, setting $x_{0}=A^{1 / 2} u_{0}$, we obtain $x_{0}+A N x_{0}=0$. Q.E.D.

THEOREM 4. Let $A: D(A) \rightarrow H$ be a selfadjoint monotone linear mapping in a real Hilbert space $H$. Let $f: H \rightarrow R$ be a weakly Gateaux differentiable functional which is also weakly-lower-semicontinuous. Let $N$ denote its weak-gradient. Assume that there is an $m>0$ such that $(N u, u) \geqq m\|u\|^{2}$ for all $u$ in $H$. Then the equation $u+A N u=0$ has at least one solution in $H$.

The proof of this theorem follows the same lines as the proof of Theorem 3.

\section{BIBLIOGRAPHY}

1. F. E. Browder, Nonlinear functional analysis and nonlinear integral equations of Hammerstein and Urysohn type, Contributions to Nonlinear Functional Analysis, Edited by E. H. Zarantonello, Academic Press, New York, 1971.

2. F. E. Browder and C. P. Gupta, Monotone operators and nonlinear integral equations of Hammerstein type, Bull. Amer. Math. Soc. 75 (1969), 1347-1353. MR 40 \#3381.

3. D. G. de Figueiredo and C. P. Gupta, Nonlinear integral equations of Hammerstein type involving unbounded monotone linear mappings, J. Math. Anal. Appl. 39 (1972), $37-48$.

4. - Nonlinear integral equations of a Hammerstein type with indefinite linear kernel in a Hilbert space, Indag. Math. 34 (1972), 335-344.

5. C. P. Gupta, On existence of solutions of nonlinear integral equations of Hammerstein type in a Banach space, J. Math. Anal. Appl. 32 (1970), 617-620. MR 42 \#3630.

6. T. Kato, Perturbation theory for linear operators, Die Grundlehren der math. Wissenschaften, Band 132, Springer-Verlag, New York, 1966. MR 34 \#3324.

7. I. M. Lavrent'ev, Solvability of nonlinear equations, Dokl. Akad. Nauk SSSR 175 (1967), 1219-1221 = Soviet Math. Dokl. 8 (1967), 993-996. MR 36 \#6990.

8. M. M. Vaĭnberg and I. M. Lavrent'ev, Equations with monotonic and potential operators in Banach spaces, Dokl. Akad. Nauk SSSR 187 (1969), 711-714=Soviet. Math. Dokl. 10 (1969), 907-910. MR 42 \#5127.

9. M. M. Vainnberg, Variational methods for the study of nonlinear operators, GITTL, Moscow, 1956; English transl., Holden-Day, San Francisco, Calif., 1964. MR 19, 567; 31 \#638.

Department of Mathematics, University of Illinois, Chicago, Illinois 60680 (Current address of D. G. deFigueiredo)

Department of Mathematics, University of Brasilia, Brasilia, Brazil

Department of Mathematics, Northern Illinois University, DeKalb, Illinois 60115 (Current address of C. P. Gupta) 